\title{
Whirling of a base-excited cantilever beam
}

\author{
Michael W. Hyer ${ }^{\text {a) }}$ \\ Department of Engineering Science and Mechanics, Virginia Polytechnic Institute and State University, \\ Blacksburg, Virginia 24061 \\ (Received 21 June 1978; revised 27 December 1978)
}

\begin{abstract}
The paper describes the response of a thin elastic cantilever beam excited by a known harmonic displacement at its base. The principal moments of the beam cross-sectional area are equal and the base excitation is in one of the principal directions. If the excitation frequency is slightly less than the resonant frequency of one of the bending modes, planar motions of the beam are unstable and an out-of-plane component of displacement develops. With this motion, the free end of the beam moves in an ellipse while the base motion is planar. Increasing the frequency to a value just above resonance causes the motion to become planar again. This whirling is a result of nonlinear coupling between the in-plane and out-of-plane displacements through the axial inertia. The paper presents the governing equations, develops the frequency-amplitude relations for the whirling response and examines the stability of the response. It is shown all whirling motions are stable and the frequency range for which whirling occurs increases with the level of excitation. Also it is shown for a given level of excitation, the frequency range broadens with increasing mode number.
\end{abstract}

PACS numbers: $43.40 . \mathrm{Cw}$

\section{INTRODUCTION}

In 1948 , in a letter to the editor, Harrison ${ }^{1}$ noted that when a thin tungsten wire, fixed at both ends, was excited by a harmonic force near the wire's resonant frequency, it was impossible to obtain planar motion. Near resonance, motions perpendicular to the plane of forcing would develop and the wire would whirl. Harrison excited the wire by passing alternating current through it and placing it in a static magnetic field. In 1960, Oplinger ${ }^{2}$ was studying the transverse motions of nylon strings by displacing one end of the string harmonically. In order to obtain planar motions, he found it necessary to constrain the string in the direction perpendicular to the direction of excitation. In 1965, Murthy and Ramakrishna ${ }^{3}$ studied this whirling phenomena. Their predictions for the in-plane and out-of-plane displacement agreed well with their experimental results. Eller ${ }^{4}$ in 1973 , in a manner similar to Oplinger, displaced the end of a string in a harmonic fashion but allowed the string to whirl. The agreement between theory and experiment was quite good. From strictly a theoretical viewpoint, Carrier, ${ }^{5,6}$ in 1945, presented the equations governing the three-dimensional motion of an elastic string. He concentrated on free-planar motions and solved the equations using perturbation techniques. However, he did outline the method for studying the nonplanar motions.

In all of these investigations, the nonlinear whirling phenomenon was a result of the string tension varying as a function of the lateral displacements. Linear theory assumes the string does not stretch and the tension is constant. This assumption is valid for small displacements, but near resonance the displacements are large and the tension change is significant. Using Hooke's Law and allowing for stretching in this string, the tension increase can be accounted for. This tension in-

\footnotetext{
2) Previously associated with the Department of Mechanical Engineering and Mechanics, Old Dominion University, Norfolk, VA 23508 .
}

crease couples the equations governing the two lateral motions, i.e., the in-plane and the out-of-plane motions, with the equation governing the axial motion. Near resonance the coupling is strong enough to cause whirling.

Continuing the theoretical investigations, Miles $^{7}$ in 1965 addressed the stability of the solutions found by Murthy and Ramakrishna. In 1966, Anand ${ }^{8}$ added damping to Murthy and Ramakrishna's equations and solved for the damped, forced response. In 1969, he addressed the stability of this response.

Compared to the work with strings, very little has been done regarding the whirling response of beams or rods. Haight and $\mathrm{King}^{10}$ studied the whirling motion of circular and nearly circular cantilever rods excited by a known harmonic displacement at their base. They predicted the excitation frequencies for which the whirling motion would occur. They found that if the excitation frequency was well below the resonant frequency of the first planar bending mode, the motion was planar. As the frequency increased, the planar displacement of course increased. However, as the excitation frequency increased to a value just below resonance, the beam would start to whirl. The whirling would continue as the excitation frequency was increased past resonance. At some value of frequency just above the planar resonant frequency, the motion would again become planar. As the frequency was increased further, the planar amplitude would decrease until the second resonant condition was approached. The whirling phenomenon would begin and end as the second resonant frequency was passed. They studied this phenomena for the first three modes and the predictions of the frequency interval for whirling agreed quite well with their experimental results. They made no attempt to study the whirling itself.

Ho, Scott, and Eisley ${ }^{11}$ did a thorough investigation of the forced response of a simply supported, axially restrained beam. T'is problem was unlike the many axi- 
ally restrained beam problems solved in the recent past (see for example Refs. 12 and 13) in that the principal moments of inertia of the beam cross section were approximately equal and out-of-plane motions were allowed. (Beams with cross-sectional areas having approximately equal principal moments of inertia are said to be compact.) The forcing was in one of the principal directions and they found as the beam approached resonance, it would whirl in a manner similar to Haight and King's cantilever rod. They predicted the in-plane and out-of-plane response and the stability characteristics of the various motions. In a later paper, ${ }^{11}$ the authors found when such a beam is given an initial lateral displacement, the beam responds with motions in the plane of the initial conditions and perpendicular to that plane.

In the axially restrained beams, the mechanism which causes the whirling is the well-known hardening spring effect of the axial restraints. Inclusion of axial force or tension, due to the axial restraint, couples the inplane and out-of-plane displacements in a manner similar to the coupling in a string.

Ultimately, in both the string and the beam, it is the use of a nonlinear strain measure which accounts for the coupling. In the beam, the bending stiffness complicates the solution to the problem. Haight and King used a nonlinear strain measure when deriving their governing equations. However, since they considered a cantilever beam, the coupling was due to axial inertia. They derived the equations governing the axial, inplane and out-of-plane motions, but only used the outof-plane equation to study the stability of the in-plane motion to out-of-plane perturbations.

It is the purpose of this paper to use their equations to investigate the whirling response of an undamped base-excited cantilever rod. The paper presents the governing equations and briefly reviews the planar solutions to those equations. The frequency-amplitude relations for the in-plane and out-of-plane components of the whirling motion are then developed. Finally, the stability of the whirling motion is studied. The paper considers only beams with equal principal crosssectional inertias, e.g., round or square cross-sectioned beams, and excitation in one of the principal directions.

\section{EQUATIONS OF MOTION}

Figure 1 illustrates the nomenclature and geometry of the base-excited cantilever. All properties are constant along the beam length. The displacements of the beam centerline in the $x, y$, and $z$ direction are given by $u$, $v$, and $w$, respectively, and the $x$ axis coincides with the undeformed centerline. The governing equations can be derived in a number of ways. Using Hamilton's principle, the bending strain energy, per unit length of beam is

$$
(E I / 2)\left[v^{\prime \prime 2}(S, t)+w^{\prime \prime 2}(S, t)\right],
$$

where $S$ is the measure of deformed arc length, ( $)^{\prime}$ denotes a partial derivative with respect to $S, E$ is Young's modulus, and $I$ is the principal moment of in-

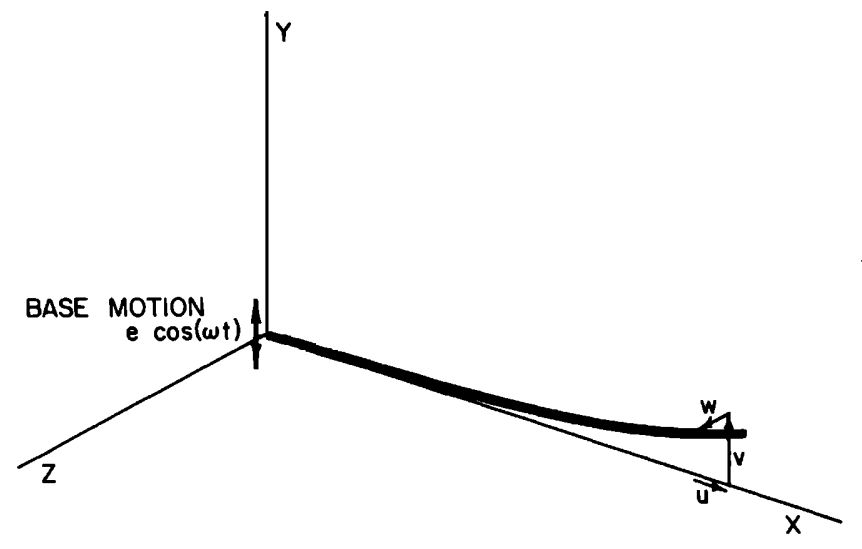

FIG. 1. Beam geometry and nomenclature.

ertia ' of the beam cross section. The axial strain energy, per unit length, is

$$
(E A / 2)\left\{u^{\prime}(S, t)+\frac{1}{2}\left[v^{\prime 2}(S, t)+w^{\prime 2}(S, t)\right]\right\}^{2},
$$

where $A$ is the beam cross-sectional area and the term in brackets is the nonlinear strain measure with $\frac{1}{2} u^{\prime 2}(S$, $t$ ) deleted. The kinetic energy, per unit length, is

$$
(m / 2)\left[\dot{u}^{2}(S, t)+\dot{v}^{2}(S, t)+\dot{w}^{2}(s, t)\right],
$$

where $m$ is mass per unit length and $\left(^{\circ}\right)$ denotes a derivative with respect to time. With this formulation, the effects of nonlinear curvature, shear deformations, twisting and rotatory inertia are ignored. Ignoring the difference between deformed and undeformed arc length, the governing equations become

$$
\begin{gathered}
-E A\left\{u^{\prime}(S, t)+\frac{1}{2}\left[v^{\prime 2}(S, t)+w^{\prime 2}(S, t)\right]\right\}^{\prime}+m \ddot{u}(S, t)=0 ; \\
E I v^{\prime \prime \prime \prime}(S, t)-E A\left\{u^{\prime}(S, t)+\frac{1}{2}\left[v^{\prime 2}(S, t)+w^{\prime 2}(S, t)\right]\right\}^{\prime} \\
v^{\prime}(S, t)-E A\left\{u^{\prime}(S, t)+\frac{1}{2}\left[v^{\prime 2}(S, t)+w^{\prime 2}(S, t)\right]\right\} \\
\times v^{\prime \prime}(S, t)+m \ddot{v}(S, t)=0 ; \\
E I w^{\prime \prime \prime \prime}(S, t)-E A\left\{u^{\prime}(S, t)+\frac{1}{2}\left[v^{\prime 2}(S, t)+w^{\prime 2}(S, t)\right]\right\}^{\prime} \\
\times w^{\prime}(S, t)-E A\left\{u^{\prime}(S, t)+\frac{1}{2}\left[v^{\prime 2}(S, t)+w^{\prime 2}(S, t)\right]\right\} \\
\times w^{\prime \prime}(S, t)+m \ddot{w}(S, t)=0 .
\end{gathered}
$$

The $v$ coordinate can be conveniently transformed by

$$
v(S, t)=\bar{v}(S, t)+e \cos \omega t,
$$

where $\bar{v}$ is the $v$ displacement over and above the rigid body effect due to base translation and $\omega$ is the excitation frequency. This transformation involves only Eq. (5) with

$$
\ddot{v}(S, t)=\ddot{\bar{v}}(S, t)-e \omega^{2} \cos \omega t .
$$

Equation (4) can be integrated once, with respect to $S$, and using the condition of no axial force on the free end of the cantilever, the integration yields

$$
E A\left\{u^{\prime}(S, t)+\frac{1}{2}\left[v^{\prime 2}(S, t)+w^{\prime 2}(S, t)\right]\right\}-m \int_{S}^{L} \ddot{u}(\xi, t) d \xi=0,
$$

where $L$ is the length of the beam. Using Eqs. (8) and (9) in Eqs. (5) and (6) and dropping the bar over the $\bar{v}$ 
gives

$$
\begin{array}{r}
E I v^{\prime \prime \prime}(S, t)-m \ddot{u}(S, t) v^{\prime}(S, t)+m\left\{\int_{S}^{L} \ddot{u}(\xi, t) d \xi\right\} v^{\prime \prime}(S, t) \\
+m \ddot{v}(S, t)-m e \omega^{2} \cos \omega t=0,
\end{array}
$$

$$
\begin{aligned}
E I w^{\prime \prime \prime \prime}(S, t)- & m \ddot{u}(S, t) w^{\prime}(S, t) \\
& +m\left\{\int_{S}^{L} \ddot{u}(\xi, t) d \xi\right\} w^{\prime \prime}(S, t)+m \ddot{w}(S, t)=0 .
\end{aligned}
$$

Finally, if the beam is assumed inextensible, the $u$ displacement can be approximated by

$$
u(S, t)=-\frac{1}{2} \int_{0}^{S}\left\{v^{\prime 2}(\xi, t)+w^{\prime 2}(\xi, t)\right\} d \xi .
$$

Using Eq. (12) in Eqs. (10) and (11) results in

$$
\begin{array}{r}
E I v^{\prime \prime \prime}(S, t)+\frac{m}{2}\left\{\frac{\partial^{2}}{\partial t^{2}} \int_{0}^{s}\left[v^{\prime 2}(\xi, t)+w^{\prime 2}(\xi, t)\right] d \xi\right\} v^{\prime}(S, t) \\
-\frac{m}{2}\left\{\int_{s}^{L}\left(\frac{\partial^{2}}{\partial t^{2}} \int_{0}^{\xi}\left[v^{\prime 2}(\eta, t)+w^{\prime 2}(\eta, t)\right] d \eta\right\} d \xi\right) v^{\prime \prime}(S, t) \\
\quad+m \ddot{v}(S, t)-m e \omega^{2} \cos \omega t=0 \\
E I w^{\prime \prime \prime \prime}(S, t)+\frac{m}{2}\left\{\frac{\partial^{2}}{\partial t^{2}} \int_{0}^{s}\left[v^{\prime 2}(\xi, t)+w^{\prime 2}(\xi, t)\right] d \xi\right\} w^{\prime}(S, t) \\
-\frac{m}{2}\left(\int_{s}^{L}\left\{\frac{\partial^{2}}{\partial t^{2}} \int_{0}^{\xi}\left[v^{\prime 2}(\eta, t)+w^{\prime 2}(\eta, t)\right] d \eta\right\} d \xi\right) \\
\times w^{\prime \prime}(S, t)+m \ddot{w}(S, t)=0 .
\end{array}
$$

Equations (13) and (14) are the equations to be solved to determine the whirling response.

\section{SOLUTION TECHNIQUE}

Since whirling occurs near the beam's resonant frequencies, the spatial shape during whirling is strongly influenced by the mode shapes of the linearized problem. Equations (13) and (14) can thus be reduced to ordinary differential equations using Galerkin's method and a one-term solution of the form

$$
\begin{aligned}
& v(S, t)=T_{2}(t) X(S), \\
& w(S, t)=T_{3}(t) X(S),
\end{aligned}
$$

where $X(S)$ is the mode shape for a cantilever beam at the resonant frequency of interest, i.e., first mode, second mode, etc. The shape is well known and is given by

$$
X(S)=\cosh \left(\beta_{n} S\right)-\cos \left(\beta_{n} S\right)-\phi_{n}\left[\sinh \left(\beta_{n} S\right)-\sin \left(\beta_{n} S\right)\right],
$$

where $\beta_{n}$ and $\phi_{n}$ depend on mode number, $n$. Equations (15) and (16) are less general than the work of Haight and King and Ho, Scott, and Eisley ${ }^{14}$ in that those investigators did not require the $v$ and $w$ displacements to have identical spatial shapes. Ho, Scott, and Eisley assumed a series solution, based on the mode shapes, and could account for possible modal interactions between the in-plane and out-of-plane displacements. Haight and King used a one-term solution for both displacements but the spatial shapes, based on the mode shapes, did
TABLE I. Coefficients associated with $n$th mode.

\begin{tabular}{cccc}
\hline$n$ & $\beta_{n} L$ & $\phi_{n}$ & $C_{n}$ \\
\hline 1 & 1.8751 & 0.7341 & 4.6 \\
2 & 4.6941 & 1.0185 & 145 \\
3 & 7.8548 & 0.9992 & 998 \\
\hline
\end{tabular}

not have to be the same. To include such effects for the cantilever, it is felt the original equations should be modified to include other effects important as modal interactions. These include the effects of nonlinear curvature, damping, shear deformation and rotatory inertia; the later three effects known to be important for higher modes. Substituting Eqs. (15) and (16) into Eqs. (13) and (14) and carrying out the integrations associated with Galerkin's method and the other spatial integrals leads to

$$
\begin{aligned}
& \ddot{\bar{T}}_{2}+\left(1 / r^{2}\right) \bar{T}+C_{n} \bar{T}_{2}\left(\bar{T}_{2} \ddot{T}_{2}+\dot{T}_{2}^{2}\right) \\
& \quad+C_{n} \bar{T}_{2}\left(\bar{T}_{3} \ddot{T}_{3}+\dot{\bar{T}}_{3}^{2}\right)=\frac{2 \bar{e} \phi_{n}}{\beta_{n} L} \cos (\tau), \\
& \ddot{T}_{3}+\left(1 / r^{2}\right) \bar{T}_{3}+C_{n} \bar{T}_{3}\left(\bar{T}_{3} \ddot{T}_{3}+\dot{T}_{3}^{2}\right)+C_{n} \bar{T}_{3}\left(\bar{T}_{2} \ddot{T}_{2}+\bar{T}_{2}^{2}\right)=0,
\end{aligned}
$$

where $\bar{T}_{2}=T_{2} / L, \bar{T}_{3}=T_{3} / L, \bar{e}=e / L, \tau=\omega t, r=\omega / \omega_{n}$. $C_{n}$ is a constant associated with the spatial integration and now $\left({ }^{\circ}\right)$ denotes a derivative with respect to time $\tau$. Table I summarizes the values of $B_{n} L, \phi_{n}$, and $C_{n}$ for the first three modes. These values are taken from Haight and King.

\section{PLANAR SOLUTION}

The planar response is found by assuming

$$
\begin{aligned}
& \bar{T}_{2}=a_{2} \cos (\tau)+b_{2} \sin (\tau), \\
& \bar{T}_{3}=0 .
\end{aligned}
$$

Substituting these into Eqs. (18) and (19) and using harmonic balance results in

$$
\begin{aligned}
& a_{2}^{3}+\left(2 / C_{n}\right)\left(1-r^{-2}\right) a_{2}+\left[4 \bar{e} \phi_{n} / C_{n}\left(\beta_{n} L\right)\right]=0, \\
& b_{2}=0 .
\end{aligned}
$$

The stability of the planar solution to in-plane perturbations is determined by substituting

$$
\begin{aligned}
& \bar{T}_{2}=a_{2} \cos (\tau)+b_{2} \sin (\tau)+\delta_{2}(\tau), \\
& \bar{T}_{3}=0
\end{aligned}
$$

into Eqs. (18) and (19), using Eqs. (22) and (23) and retaining first order terms in $\delta_{2}(\tau)$. The result is the well-known Mathieu equation and the stability boundaries, for $a_{2}<1$, are given by

$$
\begin{aligned}
& a_{2}=\left\{\left(2 / C_{n}\right)\left[\left(1 / r^{2}\right)-1\right]\right\}^{1 / 2}, r \leqslant 1 \\
& a_{2}=\left\{\left(2 / 3 C_{n}\right)\left[\left(1 / r^{2}\right)-1\right]\right\}^{1 / 2} .
\end{aligned}
$$

The stability of the solution to out-of-plane perturbations is determined by substituting

$$
\begin{aligned}
& T_{2}=a_{2} \cos (\tau)+b_{2} \sin (\tau), \\
& T_{3}=\delta_{3}(\tau)
\end{aligned}
$$

into Eqs. (18) and (19), using Eqs. (22) and (23) and re- 


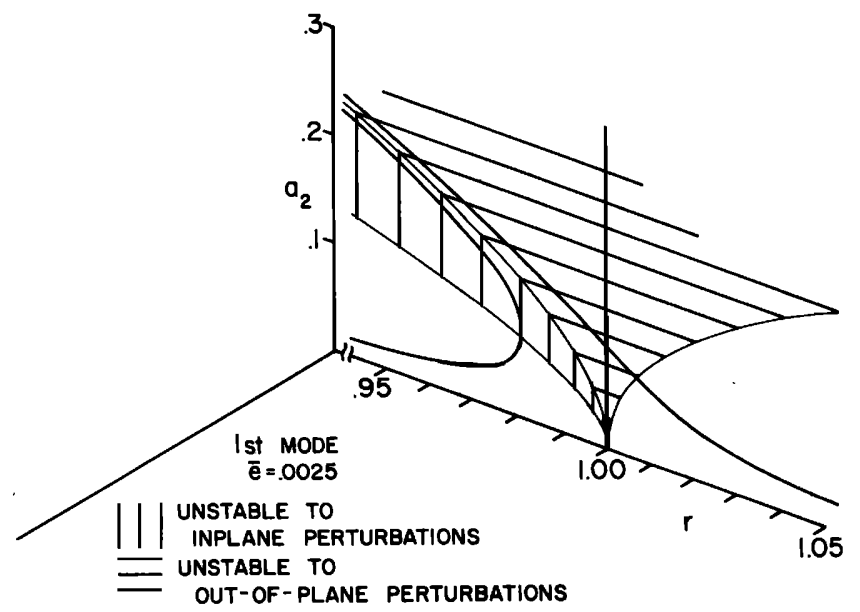

FIG. 2. Planar motion frequency response and stability characteristics.

taining first order terms in $\delta_{3}(\tau)$. The result is also the Mathieu equation and these stability boundaries, for $a_{2}<1$, are given by

$$
\begin{aligned}
& a_{2}=\left\{\left(2 / C_{n}\right)\left[\left(1 / r^{2}\right)-1\right]\right\}^{1 / 2} r \leqslant 1, \\
& a_{2}=\left\{\left(2 / C_{n}\right)\left[1-\left(1 / r^{2}\right)\right]\right\}^{1 / 2} r \geqslant 1 .
\end{aligned}
$$

The restriction $a_{2}<1$ for the stability zones results from straight line approximations, for small amplitudes, to the stability zones on the Strutt diagram associated with Mathieu's equation (See Ref. 15).

Figure 2 shows the planar response curves, in the context of potential whirling motion, for a given level of base excitation, specifically $\bar{e}=\mathbf{0 . 0 0 2 5}$. Shown are the stability zones associated with in-plane and out-ofplane disturbances. This figure represents the planar response of a $1 \mathrm{~m}$ long steel rod, $5 \mathrm{~mm}$ in diameter being excited by a displacement $1 / 2$ the beam diameter. From the figure, it is evident the governing equations represent a softening system. The unstable region associated with in-plane disturbances is bounded by the locus of vertical tangents and the free-vibration backbone curve. This region is rather well known. The unstable region associated with out-of-plane disturbances is bounded by the backbone curve and the curve $a_{2}$ $=\left\{2 / C_{n}\left[1-\left(1 / r^{2}\right)\right]\right\}^{1 / 2}$. Basically when the planar solution lies in this later region, it will move to an equilibrium solution with an out-of-plane component. This type of solution is discussed in the next section.

\section{NONPLANAR RESPONSE}

The nonplanar whirling solution is obtained by assuming

$$
\begin{aligned}
& \bar{T}_{2}=a_{2} \cos (\tau)+b_{2} \sin (\tau), \\
& \bar{T}_{3}=a_{3} \sin (\tau)+b_{3} \cos (\tau)
\end{aligned}
$$

substituting into Eqs. (18) and (19), and using harmonic balance. This results in a simple set of equations, namely

$$
\begin{aligned}
& {\left[\left(1 / r^{2}\right)-1\right] a_{2}-\frac{1}{2} c_{n} a_{2}\left(a_{2}^{2}-a_{3}^{2}\right)=2 \bar{e} \phi_{n} / \beta_{n} L,} \\
& {\left[\left(1 / r^{2}\right)-1\right] a_{3}-\frac{1}{2} C_{n} a_{3}\left(a_{3}^{2}-a_{2}^{2}\right)=0,}
\end{aligned}
$$

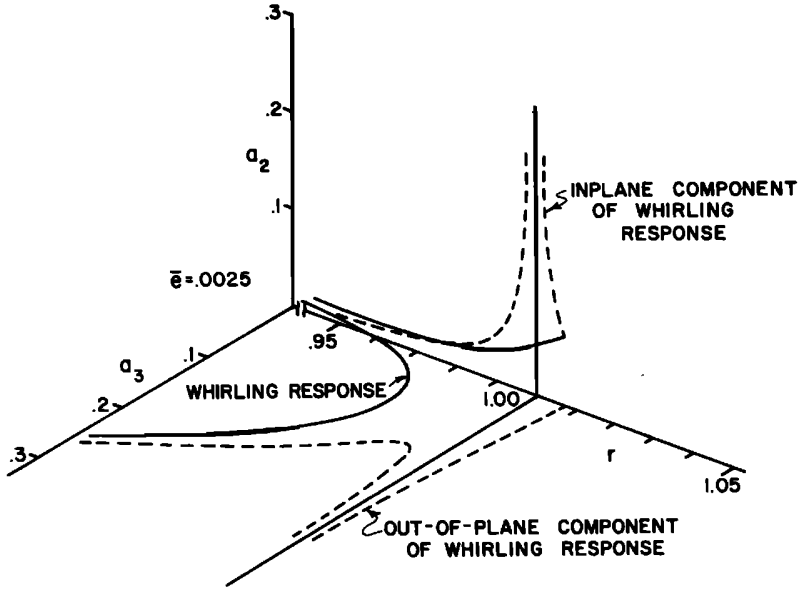

FIG. 3. Whirling motion frequency response characteristics, 1 st mode.

$$
b_{2}=b_{3}=0 \text {. }
$$

Equations (32) and (33) yield

$$
\begin{aligned}
a_{2} & =\frac{\left(\bar{e} \phi_{n} / \beta_{n} L\right) r^{2}}{1-r^{2}}, \\
\cdot a_{3} & =\left\{a_{2}^{2}+\left(2 / C_{n}\right)\left[\left(1 / r^{2}\right)-1\right]\right\}^{1 / 2} .
\end{aligned}
$$

Figures 3 and 4 illustrate the amplitude of the whirling response as a function of excitation frequency for the first two modes. The solid curves are the response curves in 3-space, while the dotted curves are the projections of the three-dimensional curves into the coordinate planes, that is, the in-plane displacement amplitude $\left(a_{2}\right)$ versus excitation frequency and the out-ofplane displacement amplitude $\left(a_{3}\right)$ versus excitation frequency relations. The characteristics for other modes and levels of excitation are similar.

Assuming the rod is whirling, say at $r=0.95$ in the first mode, the out-of-plane displacement component is larger than the in-plane component. Thus, the tip of the rod moves in an ellipse elongated in the out-ofplane direction. As the frequency increases, the out-

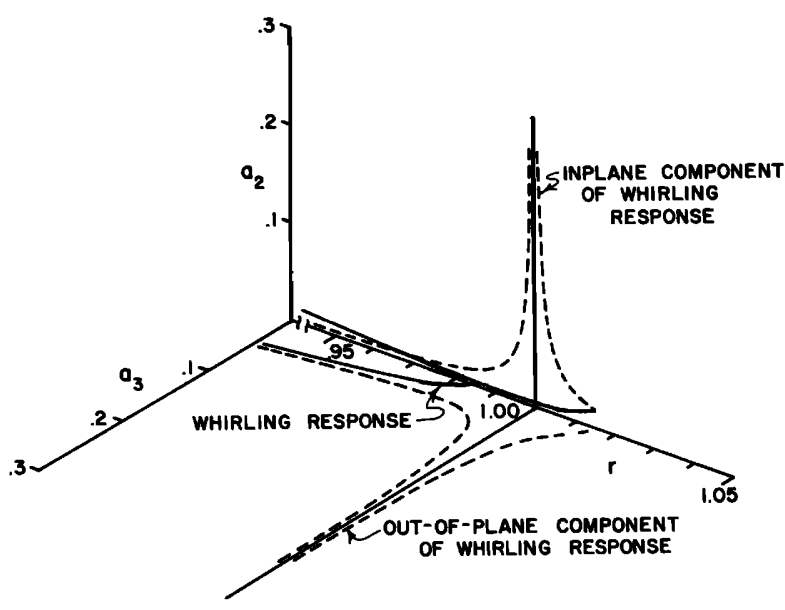

FIG. 4. Whirling motion frequency response characteristics, 2nd mode. 
of-plane component of displacement becomes smaller and the in-plane component becomes larger. As the excitation frequency is further increased, the out-ofplane component reaches a minimum value and as the frequency approaches resonance, the ellipse becomes larger and more nearly circular. When resonance is passed, a sign change in the solution indicates the direction of whirling reverses. Further increases in frequency result in a decrease in the out-of-plane amplitude. Finally, above a certain frequency, herein called the cutoff frequency, since the expression under the square root in Eq. (36) becomes negative, the whirling motion stops. For the first mode, the cutoff frequency is $r=1.007$ while for the second node it is $r=1,014$. Above the cutoff frequency, the motion is governed by the planar response solution. At the cutoff frequencies, the amplitude of the planar component of the whirling solution, Eq. (35), and the amplitude of the planar solution, Eq. (22), are so close, the jump in solutions would not be discernible.

\section{STABILITY OF WHIRLING SOLUTIONS}

As with the planar motion, first-order variational equations are used to study the stability of whirling response. Solutions of the form

$$
\begin{aligned}
& \bar{T}_{2}=a_{2} \cos (\tau)+b_{2} \sin (\tau)+\delta_{2}(\tau), \\
& \bar{T}_{3}=a_{3} \sin (\tau)+b_{3} \cos (\tau)+\delta_{3}(\tau),
\end{aligned}
$$

are substituted into Eqs. (18) and (19) and only terms to first order in $\delta_{2}$ and $\delta_{3}$ are retained. The stability equations are

$$
\begin{array}{r}
{\left[1+\frac{1}{2} C_{n} a_{2}^{2}+\frac{1}{2} C_{n} a_{2}^{2} \cos (2 \tau)\right] \ddot{\delta}_{2}(\tau)+\frac{1}{2} C_{n} a_{2} a_{3} \sin (2 \tau) \ddot{\delta}_{3}(\tau)} \\
-C_{n} a_{2} \sin (2 \tau) \dot{\delta}_{2}(\tau)+C_{n} a_{2} a_{3}[1+\cos (2 \tau)] \dot{\delta}_{3}(\tau) \\
+\left[\left(1 / r^{2}\right)-\frac{1}{2} C_{n} a_{2}^{2}+C_{n}\left(a_{3}^{2}-\frac{3}{2} a_{2}^{2}\right) \cos (2 \tau)\right] \delta_{2}(\tau) \\
-\frac{1}{2} C_{n} a_{2} a_{3} \sin (2 \tau) \delta_{3}(\tau)=0,
\end{array}
$$

$$
\begin{array}{r}
{\left[1+\frac{1}{2} C_{n} a_{3}^{2}-\frac{1}{2} C_{n} a_{3}^{2} \cos (2 \tau)\right] \ddot{\delta}_{3}(\tau)+\frac{1}{2} C_{n} a_{2} a_{3} \sin (2 \tau) \ddot{\delta}_{2}(\tau)} \\
+C_{n} a_{3}^{2} \sin (2 \tau) \dot{\delta}_{3}(\tau)-C_{n} a_{2} a_{3}[1-\cos (2 \tau)] \dot{\delta}_{2}(\tau) \\
+\left[\left(1 / r^{2}\right)-\frac{1}{2} C_{n} a_{3}^{2}+C_{n}\left(\frac{3}{2} a_{3}^{2}-a_{2}^{2}\right) \cos (2 \tau)\right] \delta_{3}(\tau) \\
-\frac{1}{2} C_{n} a_{2} a_{3} \sin (2 \tau) \delta_{2}(\tau)=0 .
\end{array}
$$

The solutions to $\delta_{2}(\tau)$ and $\delta_{3}(\tau)$ are governed by ordinary differential equations with periodic coefficients, a subject which has been well studied, (for example see Ref. 16). The solutions can of course be stable or unstable, depending on the value of the excitation frequency, $r$, the response amplitude, $a_{2}$ and $a_{3}$, and the mode, $C_{n}$. Exact solutions for Eqs. (39) and (40) exist, but in general for a given mode, the solutions divide $r-a_{2}-a_{3}$ space into regions representing stable whirling motion and regions representing unstable whirling motion. Of more concern than the solutions to Eqs. (39) and (40) are the nature of the solutions. For the problem at hand, the nature of the solution was determined in two ways. First, as an example, when studying the stability of Mathieu's equation,

$$
\ddot{x}(\tau)+[\delta+2 \epsilon \cos (2 \tau)] x(\tau)=0,
$$

where $\delta$ and $\epsilon$ are constants associated with the particular problem, the nature of periodic solutions to the equation can be used to determine the stability regions. If a region is $\delta-\epsilon$ space is bounded by periodic solutions with period $2 \pi$, the values of $\delta$ and $\epsilon$ in this region correspond to unstable motion. Based on the form of Eq. (41), the $2 \pi$ period corresponds to the principal instability. For vibratory problems, $\delta$ and $\epsilon$ are related to the amplitudes of vibration and the excitation frequency. The unstable zones in $\delta-\epsilon$ space then are mapped into amplitude-frequency space and stability is studied in terms of the amplitude and frequency. Following this idea, solutions of the form

$$
\begin{aligned}
& \delta_{2}(\tau)=C_{1} \sin (\tau)+C_{2} \cos (\tau), \\
& \delta_{3}(\tau)=C_{3} \sin (\tau)+C_{4} \cos (\tau)
\end{aligned}
$$

were sought. This form corresponds to solutions with period $2 \pi$, the principal instability for the whirling motion.

Substituting Eqs. (42) and (43) into Eqs. (39) and (40), using harmonic balance and equating the coefficients of $c_{1}, C_{2}, C_{3}$, and $C_{4}$ to zero results in the following relation between $r, C_{n}, a_{2}$, and $a_{3}$

$$
\begin{aligned}
& \left\{2\left[\left(1 / r^{2}\right)-1\right]-C_{n}\left(a_{2}^{2}+a_{3}^{2}\right)^{2}-4 C_{n}^{2} a_{2}^{2} a_{3}^{2}\right\} \\
& \times\left(\left\{2\left(1 / r^{2}\right)-1\right]+C_{n}\left(a_{3}^{2}-3 a_{2}^{2}\right)\right\} \\
& \left.\left.\quad+C_{n}\left(a_{2}^{2}-3 a_{3}^{2}\right)\right\}-4 C_{n}^{2} a_{2}^{2} a_{3}^{2}\right)=0 .
\end{aligned}
$$

For a given mode, Eq. (44) represents surfaces in $r$ $-a_{2}-a_{3}$ space. For a given value of, $a_{3}$, the unstable $r-a_{2}$ zone on the plane $a_{3}=$ constant can be determined. Using this scheme and interactive computer graphics, the nature of the three-dimensional surfaces, Eq. (44), and the regions enclosed by them can be studied.

The second method used to study stability follows the lines of Ho, Scott, and Eisley. Assuming a solution of the form

$$
\begin{aligned}
& \delta_{2}(\tau)=\left[C_{1} \sin (\tau)+C_{2} \cos (\tau)\right] e^{\lambda \tau}, \\
& \delta_{3}(\tau)=\left[C_{3} \sin (\tau)+C_{4} \cos (\tau)\right] e^{\lambda \tau} .
\end{aligned}
$$

The characteristic exponent, $\lambda$, can be used to study the stability. Substituting Eqs. (45) and (46) into Eqs. (39) and (40) using harmonic balance and setting the determinant of the coefficients for $C_{1}, C_{2}, C_{3}$, and $C_{4}$ equal to zero results in an eighth order characteristic polynomial for $\lambda$. The roots of the characteristic equation and the form of Eqs. (45) and (46) allow examination of the stability of the solutions $\delta_{2}(\tau)$ and $\delta_{3}(\tau)$ at small distances away from the surfaces given by Eq. (44). It could be argued that two approaches to studying stability are unnecessary, but owing to the amount of algebra in either approach, a check is welcome.

\section{DISCUSSION OF RESPONSE}

It should be emphasized that the complete picture of the response includes both the planar motion and the whirling motion. Figure 5 shows two solutions on the same response coordinate system. It was found to be most convenient to study the stability by looking at the stability zones for a constant $r$, thus, getting lines 


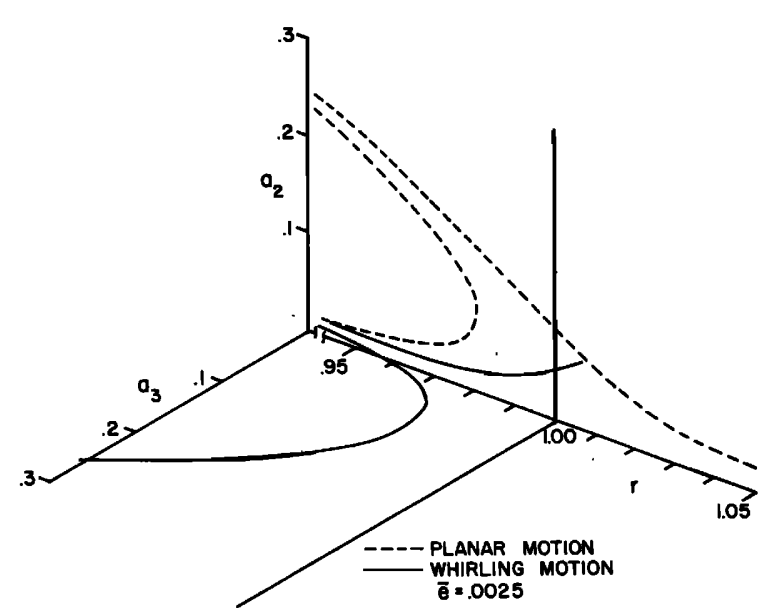

FIG. 5. Planar and whirling motion frequency response characteristics.

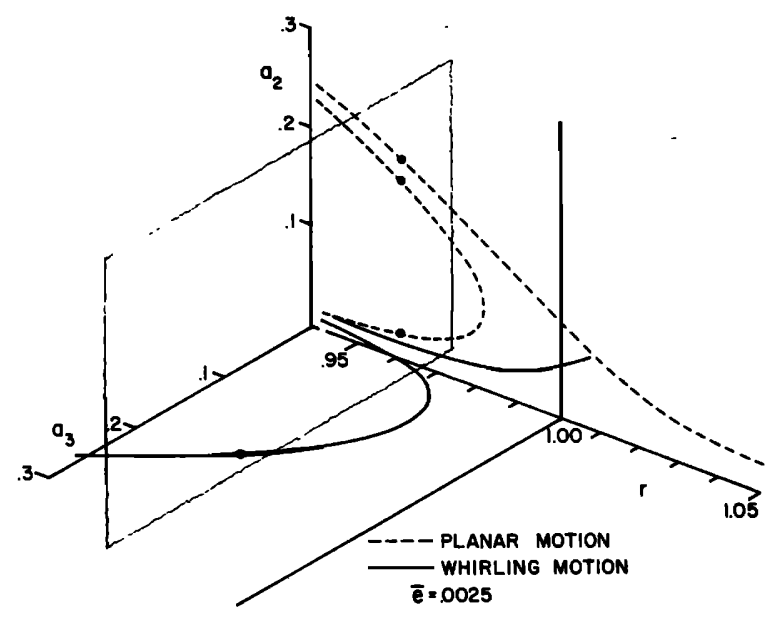

FIG. 6. Plane $r=$ constant intersecting response curves.

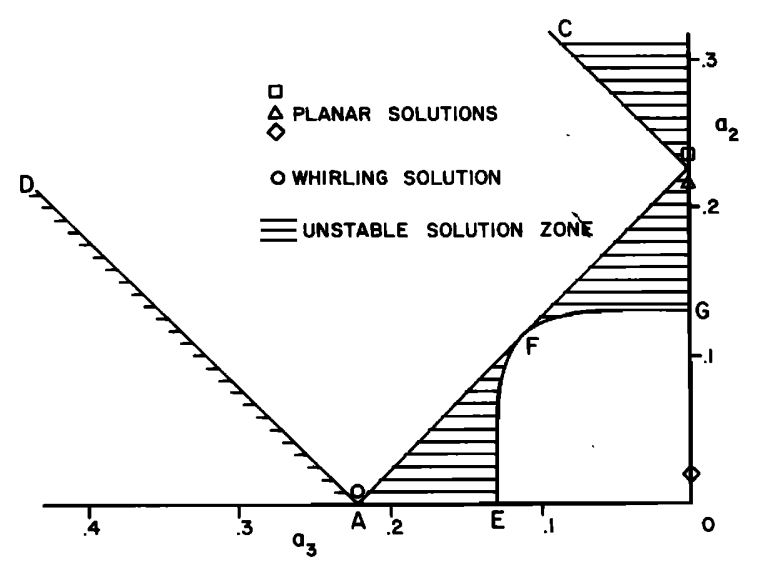

FIG. 7. Stability zones and planar and whirling solutions, 1st mode, $r=0.95, \bar{e}=0.0025$.

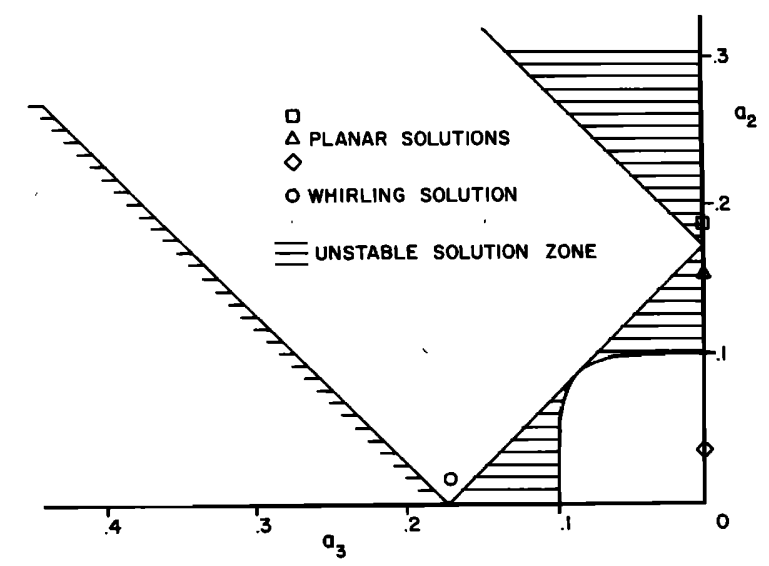

FIG. 8. Stability zones and planar and whirling solutions, 1st mode, $r=0.97, \bar{e}=0.0025$.

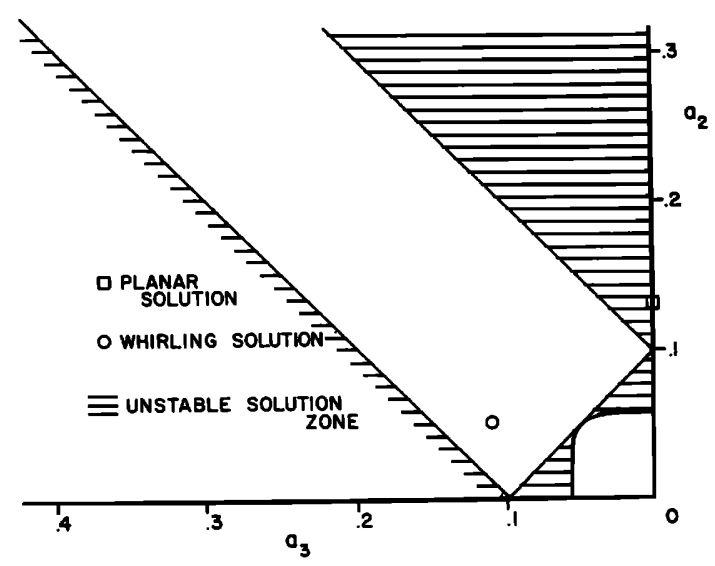

FIG. 9. Stability zones and planar and whirling solutions, 1st mode, $r=0.99, \bar{e}=0.0025$.

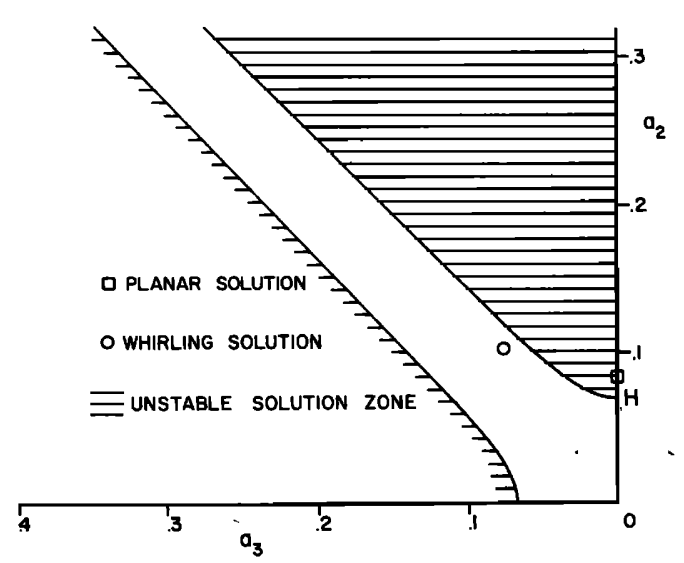

FIG. 10. Stability zones and planar and whirling solutions, 1st mode, $r=1.005, \bar{e}=0.0025$. 


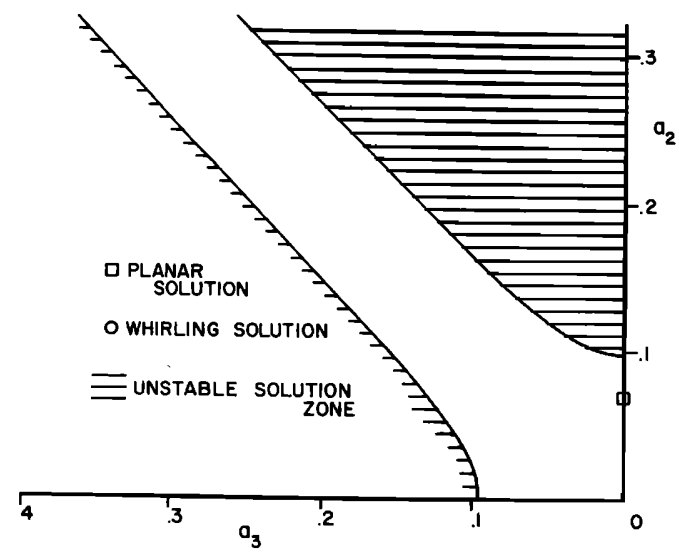

FIG. 11. Stability zones and planar and whirling solutions, 1st mode, $r=1.01, \bar{e}=0.0025$.

representing the intersection of the surfaces given by Eq. (44) with the plane $r=$ constant. In addition, on a plane $r=$ constant, the planar and nonplanar solutions are points since they are simply the intersection of the solution curves and the plane. Figure 6 depicts this idea.

Figure 7 shows the stability zones for the first mode and an excitation frequency of $r=0.95$. Lines $\mathrm{AB}, \mathrm{AD}$, $\mathrm{BC}$, and EFG are the various $a_{2}$ vs $a_{3}$ relations represented by Eq. (44) for $r=0.95$ and $C_{n}=4.6$. The one whirling solution and three' planar solutions are possible at this frequency, a frequency below the frequency corresponding to the vertical tangent of the planar response curve. One of the planar solutions is stable as is the whirling solution. The uppermost planar solution is unstable to out-of-plane perturbations, while the middle planar solution is unstable to in-plane perturbations. Depending on the initial conditions, the one stable planar solution or the whirling solution is possible. It is also possible for the actual response to jump back and forth between the two stable solutions. Point G corresponds to the intersection of the locus of vertical tangents with $r=0.95$ plane, while point $B$ corresponds to the intersection of the backbone curve with the $r$ $=0.95$ plane. The curves are symmetric about the line $a_{2}=a_{3}$. As the frequency is increased to $r=0.97$, the situation is depicted in Fig. 8. There is still one stable planar solution and the one stable whirling solution. As the frequency is increased further, the lower planar solution and middle planar solution approach each other and coalesce at the point of vertical tangency. For frequencies greater than this, say at $r=0.99$, as shown in Fig. 9, only one planar solution exists. However, this solution is unstable and the only stable solution is the whirling solution. If below the vertical tangent frequency the solution was planar, the solution would have to jump to the whirling solution as the frequency increased. If below the vertical tangent frequency the solution was nonplanar, the change in frequency would only result in
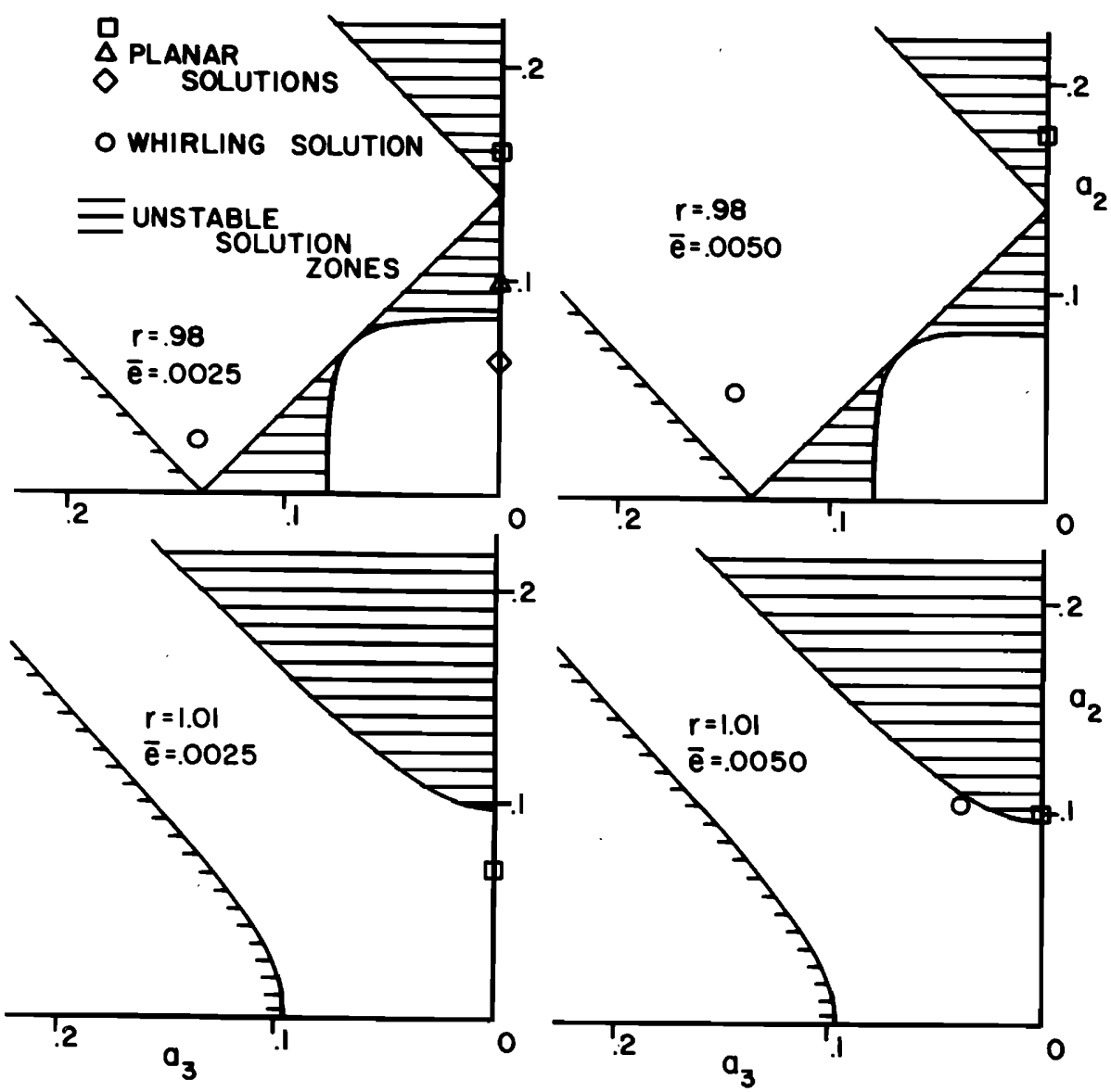

FIG. 12. Stability characteristics of the 1st mode at two levels of base excitation. 


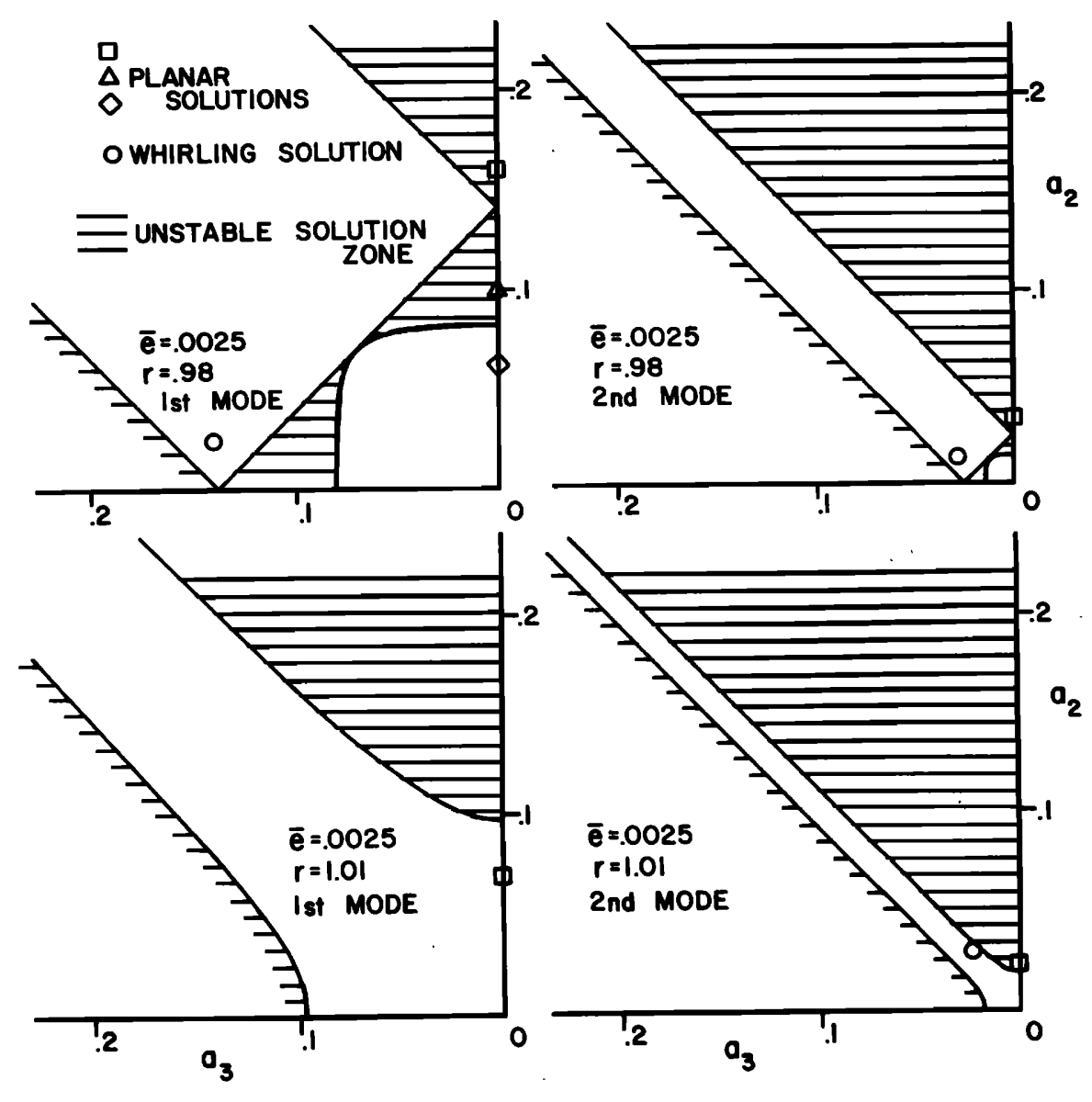

FIG. 13. Stability characteristics of the first two modes at a given level of base excitation. a change in the size of the ellipse described by the tip of the cantilever, as discussed earlier.

Past resonance, at $r=1.005$, Fig. 10, the character of the stability zones change dramatically. However, there is still one stable whirling solution and one unstable planar solution. Point $\mathrm{H}$ in Fig. 10 is the intersection of the second of Eqs. (29) with the plane $r$ $=1.005$. As the frequency is increased past the cutoff frequency, as in Fig. 11, the planar solution governs the motion.

From this example, it is clear that stable whirling occurs over a narrow frequency range near resonance. If the level of excitation is increased, the frequency range increases. Figure 12 shows the first mode stability zones for two levels of excitation, $\bar{e}=0.0025$ and $\bar{e}$ $=0.005$, at $r=0.98$ and $r=1.01$. For the higher level of excitation, since there is no stable planar solution at $r$ $=0.98$, stable whirling occurs. For the lower level of excitation, a stable planar solution exists and whirling does not necessarily occur. At $r=1.01$, a stable whirling solution is possible for the higher level of excitation while the motion is necessarily planar for the lower level. In addition, for a given level of excitation, the higher the vibration mode, the broader the frequency range over which whirling occurs. Figure 13 shows the stability zones at $r=0.98$ and $r=1.01$ for the first two modes and a given excitation level, $\bar{e}=0.0025$. At $r$ $=0.98$ a stable planar solution does not exist for the second mode while it does for the first mode. Also, for the first mode, at $r=1.01$, the motion is necessarily planar, while for the second mode stable whirling must occur.

\section{CONCLUDING REMARKS}

From the results, it can be concluded that for a baseexcited cantilever beam, stable whirling motion exists over a frequency range near the resonant frequencies of the beam. It also appears there are no unstable whirling motions in this frequency range. Whether the beam response is planar or whirling depends on the initial conditions and/or the stability of the planar solutions; however, a stable whirling solution is always possible. The effects of nonlinear curvature, damping, shear deformations, and rotatory inertia may have a bearing on the whirling response characteristics.

${ }^{1} \mathrm{H}$. Harrison, "Plane and Circular Motion of a String," J. Acoust. Soc. Am. 20, 874-875 (L) (1948).

${ }^{2} \mathrm{D}$. W. Oplinger, "F requency Response of a Nonlinear Stretched String," J. Acoust. Soc. Am. 32, 1529-1528 (1960).

${ }^{3}$ G.S.S. Murthy, and B.S. Ramakrishna, "Nonlinear Cha racter of Resonance in Stretched Strings," J. Acoust. Soc. Am., 38, 461-471 (1965).

${ }^{4}$ A. J. Eller, "Driven Nonlinear Oscillations of a String," J. Acoust. Soc. Am. 51, 960-966 (1972).

${ }^{5} \mathrm{G}$. F. Carrier, "On the Nonlinear Vibration Problem of the Elastic String," Q. Appl. Math 3, 157-165 (1945).

${ }^{6}$ G. F. Carrier, "A Note on the Vibrating String," Q. Appl. Math 7, 97 (1949). 
7J. W. Miles, "Stability of Forced Oscillations of a Vibrating String," J. Acoust. Soc. Am. 38, 855-861 (1965).

${ }^{8} \mathrm{G}$. V. Anand, "Nonlinear Resonance in Stretched Strings with Viscous Damping," J. Acoust. Soc. Am. 40, 1517-1528 (1966).

${ }^{9} \mathrm{G}$. V. Anand, "Stability of Nonlinear Oscillations of Stretched Strings," J. Acoust. Soc. Am. 46, 667-677 (1969).

${ }^{10} \mathrm{E}$. C. Haight and W. W. King, "Stability of Nonlinear Oscillations of an Elastic Rod," J. Acoust. Soc. Am. 52, 899-911 (1972).

${ }^{11}$ C. H. Ho, R. A. Scott, and J. G. Eisley, "Nonplanar, Nonlinear Oscillations of a Beam-I. Forced Motions," Int. J. Non-linear Mech. 10, 113-127 (1975).

${ }^{12}$ A. H. Nayfeh, "Nonlinear Transverse Vibrations of Beams with Properties that Vary Along the Length," J. Acoust. Soc. Am. 53, 766-770 (1973).

${ }^{13} \mathrm{~J}$. G. Eisley, "Nonlinear Deformations of Elastic Beams, Rings and Strings," Applied Mechanics Surveys, edited by H. N. Abramson (Spartan, Washington, DC, 1966), pp. 285-290.

${ }^{14}$ C. H. Ho, R. A. Scott, and J. G. Eisley, "Nonplanar, Nonlinear Oscillations of a Beam-II. Free Motions," J. Sound Vib. 47, 333-339 (1976).

${ }^{15}$ L. Meirovitch, Methods of Analytical Dynamics (McGrawHill, New York, 1970), p. 309.

${ }^{16} \mathrm{~V}$. V. Bolotin, The Dynamic Stability of Elastic Systems (Holden-Day, San F rancisco, 1964). 